\title{
Processes in Cell Ensembles: Correlated Fluctuations and their Effects
}

\author{
BY A. B. ZAHLAN \\ Physics Department, American University of Beirut, Beirut, Lebanon
}

(Received 14, May 1960)

\begin{abstract}
SUMMARY
In the study of biological systems kinetic problems arise which are not significant in the kinetic systems of interest to physical chemists. One of these biological problems is the influence of correlated fluctuations in molecular properties on the macroscopic variables of the cell. It is demonstrated that, for an ensemble consisting of systems each of which includes two chemical compounds, there is a definite effect which originates in correlated fluctuations and is reflected in a broadened distribution of the concentration of chemical constituents. It is shown that a periodically varying temperature narrows this distribution. The narrowing is a function of the frequency at which the temperature is varying.
\end{abstract}

\section{INTRODUCTION}

This study is motivated by a number of observations recorded in the literature concerning the response of cell populations to temperature variation (Zeuthen, 1958; Scherbaum, 1960). By measuring any given property of any cell in a colony, one finds that the value of that property varies from cell to cell. Rahn (1932) attempted to explain these variations by assuming a step-wise growth of the chromosomes and by attributing equal probabilities for the addition of each successive gene. He then used this treatment to investigate the dependence of the spread of division time on the number of genes. Kendall $(1948,1952)$ investigated the role of variable generation time in the development of a stochastic birth process. Kendall defines a 'multiple-phase birth process' which holds that duplication of a cell follows after the cell has passed through a finite number of phases. Both Rahn's model and Kendall's model lead to the conclusion that individual igeneration times are independent of one another. This lack of correlation of the generation times is implicit in the models.

Differences frequently exist in the properties of cells in taxonomically homogeneous colonies. A possible cause is the statistical fluctuations in the concentrations of important constituents of which each cell contains only very few molecules. We will not consider such cases in the present study.

There is no detailed experimental study of induced synchrony by a cyclic variation of temperature. The limited studies available indicate that such a procedure sometimes leads to synchrony and sometimes does not. Synchrony and spread in generation time, though perhaps interdependent in the case of a cell, are two different and distinguishable things. 


\section{Correlated fluctuations in the velocity of reactions mediated by enzymes}

Klotz and his co-workers $(1957,1958)$ have advanced the view that macromolecules are surrounded by a sheath of 'frozen water' which must be penetrated for the molecule to diffuse towards an active enzyme site. Klotz showed that if a molecule A diffuses through this sheath to the surface it must have induced some protein denaturation near the adsorption site. When molecule $\mathbf{A}$ undergoes some reaction on the surface of the enzyme it will diffuse from the site, but the succeeding molecule $\mathbf{A}^{\prime}$ diffusing to the surface will find this portion of the surface somewhat affected by the previous event. The condition of the surface is determined by random events and it is this condition which determines the reaction rate constant. Since these surface changes require a finite time, an event on the surface may influence succeeding events. Such a function of time is considered stochastic (Kittel, 1958; Uhlenbeck \& Ormstein, 1954). The statistical fluctuations in the number of hydrogen bonds lead to fluctuations in both the entropy and the energy of activation for enzyme-controlled reactions.

In order to have correlated events, the velocity of reaction $K(t+\tau)$ associated with an enzyme $\mathbf{E}$ at time $(t+\tau)$ must be related to the velocity of reaction $K(t)$ at time $t$. This is possible when a reacting molecule diffuses to the reaction site and completes its chemical transformation in a time roughly equal to the correlation time of the function $K(t)$. Consider an enzyme $\mathbf{E}$ on whose surface the compounds $A$ and $B$ react at two different sites. If there is energy or charge transfer between the reacting sites (cf. Szent-Gyorgyi, 1957) then these reactions are coupled. The rate of one reaction is then controlled by the rate of the other reaction and the condition of the surface. These processes are stochastic in character and lead to correlated fluctuations. Our model of the cell is then one where thousands of chemical reactions are taking place, each of which is influenced by its environment in the manner discussed above. Every so often the cell duplicates itself and breaks up into two new cells. Since it is the cumulative effect of all of these reactions that leads to cell division it is clear that any fluctuations in these reactions will be reflected in the cell division time. That is, the cells of a population will not all have the same generation time (time between two divisions).

In this study we wish to demonstrate the role of correlated fluctuations in dephasing synchronized colonies and to show how a cyclic variation in temperature 'damps' such correlation effects. The total effect of temperature variation (specially when $T$ is large) is more complex than is assumed here. A current study (by author, to be published) analyses these phenomena.

\section{The treatment of a simple model which displays correlated fluctuations}

Constant temperature. To demonstrate the validity of our model we have to write the equations which describe the space-time variations of all the chemical species in a cell, allow for diffusion of material across the cell wall, then let the rate constants vary in a stochastic manner and show that the cumulative effect of these correlated fluctuations in the rate constants leads to a broadening in the spectral distribution of various cell properties. At present it is impossible to carry out such a complete programme. Instead, in the following sections we shall analyse the simplest example of such a kinetic system, namely, an ensemble of systems each of which is 
composed of two compounds $(1,2)$, each of which is changed into the other pseudounimolecularly:

$$
\mathrm{E}_{1}+1 \rightarrow 2 ; \quad \mathrm{E}_{2}+2 \rightarrow 1 .
$$

We shall assume that these processes are controlled by two different enzymes and that because of environmental effects, the rate constants are time dependent and stochastically correlated.

We shall compute the first and second moments of the distribution of the number of molecules of each compound in the ensemble. This is simpler than finding the complete distribution function for each compound over the whole ensemble. The second moment shows the 'broadening' in the distribution function due to these random variations, in whose absence there would be no dispersion. When the temperature is made to vary periodically with time the distribution narrows; i.e. the second moment is diminished.

The two compounds are now allowed to be transformed pseudo-unimolecularly into each other at the rates $k_{12}(t), k_{21}(t)$, where the $k(t)$ 's are stochastic variables dependent on a catalytic process. Let $p_{1}, p_{2}$ be the mole fractions of each species; we have then

Define

$$
\begin{aligned}
& d p_{1} / d t=k_{12} p_{2}-k_{21} p_{1}, \\
& d p_{2} / d t=k_{21} p_{1}-k_{12} p_{2} .
\end{aligned}
$$

We now analyse the rate constants into Fourier series:

$$
\begin{aligned}
& k_{12}(t)=k_{12}^{0}+\sum_{n}\left(A_{n} \sin n t+B_{n} \cos n t\right), \\
& k_{21}(t)=k_{21}^{0}+\sum_{n}\left(C_{n} \sin n t+D_{n} \cos n t\right),
\end{aligned}
$$

where $k_{12}^{0}$ and $k_{21}^{0}$ are average values of the rate constants and $\left(A_{n}, B_{n}, C_{n}, D_{n}\right)$ are all randomly distributed functions. We now assume that the random variable components average to zero within the time interval $0 \rightarrow t$ of interest to us, but not within times of the order of milliseconds. This means that a molecule could diffuse to a catalytic surface and depart from such a location within a time interval short compared to the duration of our experiment or to the rate of variation of the condition of the catalytic surface. This assumption simplifies our problem considerably, since now we can set

$$
1 / t \int_{0}^{t} \lambda d t=k_{12}^{0}+k_{21}^{0}=\lambda^{0}
$$

and our solution becomes:

$$
p_{1}(t)=p_{1}(0) e^{-\lambda^{0} t}+e^{-\lambda^{0} t} \int k_{12} e^{\lambda^{0} t} d t
$$

Let us now average over the ensemble $A$.

but

$$
\therefore\left\langle p_{1}\right\rangle_{\Delta}=\left\langle p_{1}(0)\right\rangle_{A} e^{-\lambda^{0} t}+e^{-\lambda^{0} t} \int e^{\lambda^{0} t}\left\langle k_{12}\right\rangle_{A} d t,
$$

Since ensemble averages and time averages may be assumed to lead to the same results,

$$
\lim _{t \rightarrow \infty}\left\langle p_{1}\right\rangle_{\Delta}=k_{12}^{0} / \lambda^{0} \text {. }
$$


Now, instead of specifying the random variables $\left(A_{n}, B_{n}, C_{n}, D_{n}\right)$ in more detail (such as a set of Gaussianly distributed functions) we follow Uhlenbeck \& Ormstein's (1954) procedure and simply seek the second moment about the origin $\left\langle p_{1}^{2}\right\rangle_{\Delta}$.

On squaring the solution for $p_{1}(t)$ and averaging over the ensemble $\mathbf{A}$, we find

One then finds

$$
\left\langle p_{1}^{2}\right\rangle_{A}=\left\langle p_{1}^{2}(0)\right\rangle_{\Delta} e^{-2 \lambda^{0} t}+e^{-2 \lambda^{0} t} \iint e^{\lambda(\xi+\eta)}\left\langle k_{12}(\xi) k_{12}(\eta)\right\rangle_{A} d \xi d \eta
$$

$$
\begin{aligned}
\left\langle p_{1}^{2}\right\rangle_{\Delta}=\left\langle p_{1}^{2}(0)\right\rangle_{\Delta} e^{-2 \lambda^{0} t}+ & \frac{\left(k_{12}^{0}\right)^{2}}{2 \lambda^{0} \tau_{1}}\left(1-e^{-2 \lambda^{0} t}\right) \\
& +\left(\frac{k_{12}^{0}}{\lambda^{0}}\right)^{2}\left(1-e^{-\lambda^{0} t}\right)-\left(\frac{k_{12}^{0}}{\lambda^{0}}\right)\left(1-e^{-\lambda^{0} t}\right) e^{-\lambda^{0} t}
\end{aligned}
$$

and

$$
\tau_{\mathbf{1}}^{-1}=\int_{-\infty}^{\infty} \phi(w) d w
$$

where $\phi(w)$ is the correlation function, where $w=\xi-\eta$. Then

$$
\begin{aligned}
\lim _{t \rightarrow \infty}\left\langle p_{1}^{2}\right\rangle_{A} & =\left(k_{12}^{0} / \lambda^{0}\right)^{2}+\left(k_{12}^{0}\right)^{2} / 2 \lambda^{0} \tau_{1} \\
& =\left\langle p_{1,0}^{2}\right\rangle_{A} .
\end{aligned}
$$

The significance of this result is that in a system where correlation effects are absent we should have

$$
\left\langle p_{1}^{2}\right\rangle_{\Delta}=\left\langle p_{1}\right\rangle_{A}^{2}
$$

Here, we have a broadened density function; the second moment is increased by

$$
\left(k_{12}^{0}\right)^{2} /\left(2 \lambda^{0} \tau_{1}\right) \text {. }
$$

This is the variance of the distribution.

Let

$$
\text { The effect of a sinusoidal variation of temperature }
$$

$T$ is about $300^{\circ} \mathrm{K}$. in most biological reactions while $B$ is about $10^{\circ} \mathrm{C}$.; therefore, the ratio $T / B$ is about 30 . One can then write

where

$$
A e^{-R \mid R T} \simeq k\left(T_{0}\right)\left[1+\frac{E B \cos f t}{R T_{0}^{2}}\right]
$$

$$
k\left(T_{0}\right)=A e^{-E R T_{0}} \text {. }
$$

This time variation of the temperature does not affect $\left\langle p_{1}\right\rangle_{A}$. However $\left\langle p_{1}^{2}\right\rangle_{A}$ changes to

where $\tau_{1}$ is defined as before.

$$
\begin{aligned}
\lim _{t \rightarrow \infty}\left\langle p_{1}^{2}\right\rangle_{\Lambda}=\left(\frac{k_{12}^{0}}{\lambda^{0}}\right)^{2} & +\left(\frac{k_{12}^{0}}{2 \lambda^{0} \tau_{1}}\right)-\frac{2 \lambda^{0} f}{f^{2}+\tau_{13}^{2}}\left(\frac{B A_{12}^{0} G_{12}^{0}}{R T_{0}^{2} \lambda^{0}}\right)^{2} \\
& +\frac{B A_{12}^{0} G_{12}^{0} \lambda^{0}}{R T_{0}^{2} \tau_{13}\left(\lambda^{02}-f^{2}\right)}\left[\sin 2 f t-\left(\frac{f}{\lambda^{0}}\right) \cos 2 f t\right]
\end{aligned}
$$

But

$$
\tau_{13}=\tau_{11}+\tau_{12}
$$

and $\tau_{11}, \tau_{12}$ are defined by the correlation functions:

$$
\begin{gathered}
\left\langle A_{12}(\xi) A_{12}(\eta)\right\rangle=\left\langle A_{12}^{2}\right\rangle e^{-\tau_{11} w}=\left(A_{12}\right)^{2} e^{-\tau_{11} w} \\
\left\langle E_{12}(\xi) e^{-E_{12}(\xi) / R T_{\bullet}} \cdot E_{12}(\eta) e^{-E_{13}(\eta) / R T_{\bullet}}\right\rangle=\left(G_{12}^{0}\right)^{2} e^{-\tau_{12} w}
\end{gathered}
$$


We note the existence of a 'forced motion' term $\left\{\sin 2 f t-\left(f / \lambda^{0}\right) \cos 2 f t\right\}$ due to the periodic variation in the temperature. We can eliminate this term by taking a time average over one cycle.

where

$$
\left\langle\lim _{t \rightarrow \infty}\left\langle p_{1}^{2}\right\rangle_{\Delta}\right\rangle_{\text {one cycle }}=\left\langle p_{1,0}^{2}\right\rangle_{\Delta}-\frac{c f}{\tau_{13}^{2}+f^{2}},
$$

$$
C=\left(2 / \lambda^{0}\right)\left(A_{12}^{0} G_{12}^{0} B / R T_{0}^{2}\right)^{2} .
$$

Equation (23) displays a reduction in the variance induced by temperature variation. We will now give a brief analysis of this important result. The distribution will be narrowest for $f=\tau_{13}$. One can show that for all reasonable values of the constants involved the second moment will have a value intermediate between the case where no correlated fluctuations exist and where $f=0$. Note that the narrowing goes to zero as $f$ or $B$ go to zero. The narrowing is a function of the parameters of the system, such as $\tau_{11}, \tau_{12}, \tau_{13}, A$ and $G$. This is why this approach might be fruitful in investigating complex molecular systems.

\section{Generalization of the results for a specialized many component system}

So far we have considered an ensemble of two enzyme systems (or cells). We now generalize these results to an ensemble of $N$-component systems. Each $N$-component system is now referred to as a 'cell' and the ensemble of such cells is referred to as a colony of cells. That is, if

where

$$
\begin{gathered}
p=\left(p_{1}, p_{2}, \ldots, p_{N}\right) \\
\sum_{i=1}^{N} p_{i}=1 \\
\frac{d}{d t} \mathrm{p}(t)=\left(\begin{array}{ccc}
k_{11} & \ldots & k_{1 N} \\
\vdots & & \\
k_{N 1} & \ldots & k_{N N}
\end{array}\right)\left(\begin{array}{cc}
p_{1} & (t) \\
\vdots & \\
p & (t)
\end{array}\right)
\end{gathered}
$$

Let every cell in the colony be denoted by a superscript. Then $\boldsymbol{P}^{a}(t)$ denotes the composition of the ath cell at time $t$. At any time $t$, one can determine the average molar concentration of any species ( $i$, say) to be

$$
p_{0 i}(t)=1 / M \sum_{a=1}^{M} p_{i}^{a}(t) \quad(\boldsymbol{M}=\text { no. of cells in colony })
$$

The lack of uniformity (synchrony) in the colony can be indicated by

$$
\Delta_{i}(t)=\left\langle\left|\boldsymbol{P}_{i}(t)-\boldsymbol{P}_{0 i}(t)\right|\right\rangle_{a},
$$

and

$$
d^{2}=\left\langle\overline{P_{i}-P_{0 i}^{2}}\right\rangle_{a} \text {. }
$$

As before, we want to show that the correlated fluctuations in the $k_{i j}$ 's lead to an increase in the value of the second moment $d^{2}(t)$ and that a periodic temperature variation leads to a narrowing in the distribution, i.e. a decrease in the value of $d_{i}^{2}(t)$. The solution (Bellman, 1953) of such a system of first-order differential equation is of the type

$$
\mathbf{p}(t)=\mathbf{C Y}(t) e^{B t},
$$

where $C$ is a constant matrix given by $P(0)=C$, and $Y(t)$ contains the timedependent portion.

Comparing this equation with equation (8) one can observe the features necessary to contribute to the broadening of $\mathrm{p}(t)^{2}$ due to $\left\langle[Y(t)]^{2}\right\rangle_{a}$ being broadened by correlated fluctuations in the same sense as $k_{12}$ though this result is more complicated. 
Similarly, temperature variation will induce a decrease in the value of $\left\langle[Y(t)]^{2}\right\rangle_{a}$. This argument is naturally not rigorous. The more general solution of the problem will be postponed for a later publication.

\section{Synchronization of cell colonies by periodic variation of light intensity}

Zeuthen (1958) reviewed experimental observations on colony synchronization by periodic variation of light intensity. It is simple to reduce this problem to the one already discussed above. Consider the same ensemble of two-component systems except that the processes are now both catalytic and photochemical, i.e.

Then

$$
\begin{aligned}
& E_{1}+h \nu_{1}+(1) \stackrel{a_{12}}{\rightarrow}(2), \\
& E_{2}+h v_{2}+(2) \stackrel{a_{31}}{\rightarrow}(1) .
\end{aligned}
$$

therefore

$$
\begin{gathered}
d P_{2} / d t=a_{12} p_{1} I_{1}-a_{21} p_{2} I_{2}, \\
p_{1}+p_{2}=1,
\end{gathered}
$$

$$
d p_{1} / d t=a_{21} I_{2}-\lambda p_{1} \text {. }
$$

If $I_{1}$ and $I_{2}$ are constant we find

$$
p_{1}=p_{1}(0) \exp \left[-\int \lambda d t\right]+\exp \left[-\int \lambda d t\right] \int\left[a_{12} I_{2} \exp \left[\int \lambda d t\right]\right] d t
$$

which is identical in form with equation (8). However, if $\left(I_{1}, I_{2}\right)$ are time-dependent we obtain an equation similar to the one we were led to through equation (18); except for minor changes in the parameters involved in our equations $(23,24)$ we obtain similar results as before.

\section{REFERENCES}

Bellman, R. (1953). Stability Theory of Differential Equations. New York: McGraw Hill Book Co. Inc.

KendaLL, D. G. (1948). On the role of variable generation time in the development of a stochastic birth process. Biometrika, 35, 316.

Kendall, D. G. (1952). On the choice of a mathematical model to represent normal bacterial growth. J. R. statist. Soc. B, 14, 41.

KrTtel, C. (1958). Elementary Statistical Physics, pp. 118-68. New York: J. Wiley and Sons Inc.

KLOtz, I. M. \& HeIney, R. E. (1957). Changes in protein topography upon oxygenation, Proc. nat. Acad. Sci., Wash. 47, 717.

KLOTz, I. M. \& AyERs, J. (1957). The hydrogen ion equilibria of a single group attached to serum albumin: some implications as to surface characteristics of protein molecules, J. Amer. chem. Soc. 79, 4078.

Kцотz, I. M. (1958). Protein hydration and behaviour. Science, 128, 815.

RAHN, O. (1932). A chemical explanation of the variability of the growth rate. J. gen. Physiol. 15, 257.

Scherbaum, O. (1960). Synchronous division of microorganisms. Annu. Rev. Microbiol. $14,238$.

Szent-Gyorgyi, A. (1957). Bioenergetics. New York: Academic Press Inc.

Uhlenbeck, G. E. \& Ormstern, L. S. (1954). Noise and Stochastic Processes. Ed. by N. Wax, New York: Academic Press Inc.

Zeuthen, E. (1958). Artificial and induced periodicity in living cells. Advances in Biological and Medical Physics, 6, p. 37. New York: Academic Press Inc. 